WM-00-105

\title{
Predictions for Decays of Radially Excited Baryons
}

\author{
Carl E. Carlson* and Christopher D. Carone ${ }^{\dagger}$ \\ Nuclear and Particle Theory Group, Department of Physics, College of William and Mary, \\ Williamsburg, VA 23187-8795
}

(May, 2000)

\begin{abstract}
We consider the strong decays of the lowest-lying radially excited baryons, in SU(6) language the states comprising the first excited 56-plet. Assuming a single-quark decay approximation, and negligible configuration mixing, we make model-independent predictions for the partial decay widths to final states with a single meson. Masses of unobserved states are predicted using results from large- $N_{c}$ QCD, and the momentum dependence of the one-body decay amplitude is determined phenomenologically by fitting to observed decays, so that the baryon spatial wave functions are not assumed. We point out that comparison of these predictions to experiment may shed light on whether the Roper resonance can be consistently interpreted as a three-quark state.
\end{abstract}

\footnotetext{
*carlson@physics.wm.edu

${ }^{\dagger}$ carone@physics.wm.edu
} 


\section{INTRODUCTION}

In this note, we consider the strong decays of the lowest-lying radially excited baryons. These are the states comprising the first excited 56-plet, in SU(6) language. We make predictions for the decay widths of these states that are independent of any specific model for the binding potential or spatial wave functions.

A reason for taking up studies of excited baryons now is that we anticipate new results from the CLAS Collaboration at the Thomas Jefferson National Accelerator Facility. Not all the states in the radially excited 56-plet are yet discovered, and many of the measured decay widths of the observed states have large uncertainties. A set of predictions for the decay widths of unobserved states will be of great use to experimenters. Of necessity, we also provide predictions for the masses of the unobserved states. To this end, we present a derivation of the Gürsey-Radicati mass formula [1] from large- $N_{c}$ QCD that we believe has not appeared explicitly in the literature.

Another and more specific reason for our analysis is to shed light on the Roper resonance, the $N(1440)$. The Roper is the first excitation of the nucleon with the same $J^{P}=(1 / 2)^{+}$ quantum numbers. The most direct explanation of the Roper is as a three-quark radially excited state. However, certain of its apparent peculiarities have led to plausible suggestions that it may be a hybrid state [0] (a state whose lowest significant Fock component is three quarks plus gluonic excitations) or that it may be a cross section enhancement that does not correspond to any resonance [3]. An argument against interpreting the Roper as a three-quark state has been that its calculated mass is too high in quark models with one-gluon-exchange, i.e. spin-color, interactions [4]. Recently, it has been found that the observed mass is perfectly consistent with the three-quark picture if mass splittings are due to spin-flavor interactions instead [5]. Moreover, there is evidence of a Roper signal from a lattice calculation using a three-quark source operator, although uncertainties in the mass determination as yet preclude saying if it is lighter than its negative parity counterpart [6]. In the present work, we assume the Roper resonance is a three-quark state embedded in the excited 56-plet; confirmation of the decay widths predicted here will therefore support the three-quark interpretation of this state.

The value of a model-independent analysis should be clear: We are free of any assumptions regarding the binding potential or quark spatial wave functions. We do make assumptions about the dominance of single-quark operators and about configuration mixing that we now consider in turn.

First, we assume that the decays of interest proceed via a single quark interaction vertex. We may write the decay operator in the same $\mathrm{SU}(6)$ tensor product space in which we define the baryon spin-flavor wave functions:

$$
\mathcal{H}_{\text {eff }} \propto G_{*}^{i a} k^{i} \pi^{a}
$$

Here $G_{*}^{i a}=S_{*}^{i} T_{*}^{a}$ is an $\mathrm{SU}(6)$ generator, where $S_{*}^{i}$ and $T_{*}^{a}$ are the generators of $\mathrm{SU}(2)$ and $\mathrm{SU}(3)$, respectively, $k^{i}$ is the meson three-momentum, and $\pi^{a}$ represents a meson field operator. The asterisk in Eq. (1.1) indicates that that the operator acts on the spin-flavor wave function of the excited quark. Here we adopt a convenient picture of the baryon state that follows from large- $N_{c}$ QCD, namely, that the baryon consists of a 'core' of $N_{c}-1$ ground state quarks generating a collective potential in which a single quark is excited. Note that 
the complete symmetry of the 56-plet spatial wave functions implies that matrix elements of the operator in Eq. (1.1) are proportional to matrix elements of the same spin-flavor operator summed over all the quarks in the baryon state,

$$
G^{i a} \equiv \sum_{\text {quarks } \alpha} S_{\alpha}^{i} T_{\alpha}^{a}
$$

Thus, the operator in Eq. (1.1) works equally well in parameterizing the decays of radially excited baryons for $N_{c}=3$, where the state may be described more realistically as a collective excitation of all three quarks. Matrix elements of this operator may be written

$$
\left\langle\Psi\left(B_{f}, \pi^{a}\right)\left|\mathcal{H}_{\mathrm{eff}}\right| \Psi\left(B_{i}\right)\right\rangle=f(k) k^{j}\left\langle B_{f}\left|G^{j a}\right| B_{i}\right\rangle
$$

where $k=|\vec{k}|$ and $f(k)$ is a function that parameterizes the momentum dependence of the amplitude. This includes momentum dependence that originates from the underlying quarklevel vertex, as well as from the overlap of the baryon spatial wave functions. The baryon states shown represent only the spin-flavor part of the wave function, and are nonrelativistically normalized. Use of the single-body decay approximation is strongly motivated by its success in describing decays of the orbitally excited, 70-plet baryons, both in the algebraic $\mathrm{SU}(6)$ approach [7], as well as more recent large- $N_{c}$ effective field theory analyses [8 11]. In the latter case, both one- and two-body operators have been shown to contribute to 70-plet decay matrix elements at leading order in the $1 / N_{c}$ expansion. Nonetheless, comparison to data for both strong decays [9] and photoproduction amplitudes [10] reveals that the twobody operators are phenomenologically irrelevant. It is not unreasonable to assume that similar dynamics may play a role in the strong decays of other excited SU(6) multiplets.

Our second assumption is that mixing between states in the excited 56-plet and other $\mathrm{SU}(6)$ multiplets, i.e. configuration mixing, can be neglected. In the case of the 70-plet, successful large- $N_{c}$ effective theory descriptions of the strong decays [9], photoproduction amplitudes [10], and the nonstrange mass spectrum [8] all neglect mixing with other SU(6) multiplets. In the present case, one might worry that the nearest observed states with appropriate quantum numbers for mixing, members of the positive-parity 70-plet, are only between 200 and $300 \mathrm{MeV}$ heavier than the states of interest, so that a significant effect cannot be precluded. Fortunately, evidence from explicit quark models suggests that configuration mixing involving the excited $\mathbf{5 6}$ is also small [12], and even smaller than one would expect from $1 / N_{c}$ arguments [13]. We simply adopt this as a working assumption, without wedding ourselves to a particular quark model or choice of baryon spatial wave function.

\section{ANALYSIS OF OBSERVED STATES}

We first determine the functional form and normalization of $f(k)$ by considering the decay modes of observed members of the excited 56-plet, the $N(1440), \Delta(1600), \Lambda(1600)$ and the $\Sigma(1660)$. We will refer to this multiplet as the $\mathbf{5 6}^{\prime}$ henceforth. (The RPP refers to

\footnotetext{
${ }^{1}$ Note that the suppression of two-body operators in the present case implies that F-wave decay amplitudes should be highly suppressed.
} 
the $\mathbf{5 6 ^ { \prime }}$ as the $\left(56,0_{2}^{+}\right)$.) Our input values are shown in Table \&, and have been extracted from data in the Review of Particle Physics (RPP) [14. The one standard deviation errors on the baryon masses were taken to be half of the corresponding mass ranges given in the RPP, and partial widths were computed using the quoted full widths, branching fractions, and associated errors. The values for $f(k)$ corresponding to each observed decay mode are shown in Fig. 1. Since we wish to model the function $f(k)$ for momenta from $\sim 100$ to $\sim 500 \mathrm{MeV}$, we require only that we find a simple functional form that works well within this range. The most successful result is $f(k)=(2.8 \pm 0.2) / k$, which has a $\chi^{2}$ per degree of freedom of 1.1. Note that the functional forms $a+k b, a / k^{0.5}, a / k^{1.5}$ lead to a $\chi^{2}$ per degree of freedom of $1.9,1.6$, and 2.8 , respectively. It is somewhat remarkable that such a simple functional form can account for the data in the momentum range of interest, though admittedly, the experimental uncertainties are large. It is not inconceivable, for example, that improvement in the data could lead to a preference for the less singular-looking linear fit, but given the present errors, the difference between a linear and the $1 / k$ fit makes little difference in our decay predictions. Continuing in our spirit of model independence, we do not try to ascertain the origin of $f(k)$, but explore how far we can go in predicting decay modes for unobserved states.

\section{MASS PREDICTIONS}

We use the Gürsey-Radicati (GR) formula to predict masses for the unobserved members of the 56-plet:

$$
M=A+B N_{s}+C\left[I(I+1)-N_{s}^{2} / 4\right]+D J(J+1) .
$$

Here $\mathrm{J}(\mathrm{I})$ is the baryon spin (isospin) and $N_{s}$ is the number of valence strange quarks. The GR formula predicts spin- and flavor-dependent mass splitting for completely symmetric $\mathrm{SU}(6)$ baryon multiplets in terms of the four parameters $A, B, C$ and $D$; in our case,

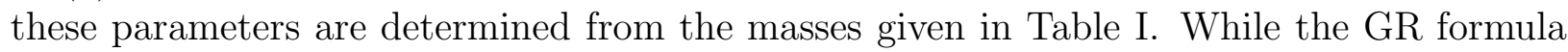
was originally a conjecture [1], and later 'derived' by assuming SU(6) symmetry and a specific set of mass operators transforming in symmetry-breaking representations of small dimensionality [15], we begin by showing how Eq. (3.1) may be obtained more rigorously from large- $N_{c}$ QCD. This observation follows from the discussion in Ref. [16], but is not presented there explicitly. In a large- $N_{c}$ operator analysis for the $\mathbf{5 6}$-plet, we may write the general mass formula

$$
\begin{aligned}
M & =a_{1} \mathbb{1}+a_{2} \frac{S^{2}}{N_{c}}+\epsilon a_{3} T^{8}+\epsilon a_{4} \frac{S^{i} G^{i 8}}{N_{c}}++\epsilon a_{5} \frac{S^{2} T^{8}}{N_{c}^{2}}+\epsilon^{2} a_{6} \frac{T^{8} T^{8}}{N_{c}} \\
& +\epsilon^{2} a_{7} \frac{T^{8} S^{i} G^{i 8}}{N_{c}^{2}}+\epsilon^{3} a_{8} \frac{T^{8} T^{8} T^{8}}{N_{c}^{2}},
\end{aligned}
$$

where $S, T$, and $G$ are the spin, flavor, and spin-flavor generators of SU(6) (with a sum over quarks left implicit), and $\epsilon \sim 1 / 3 \sim 1 / N_{c}$ parameterizes the size of $\mathrm{SU}(3)$ breaking. Notice that the eight order-one coefficients, $a_{1} \ldots a_{8}$, completely span the space of observables, namely the eight baryon mass eigenvalues. Assuming that the baryon states have spin, isospin, and strangeness of order one in the large $N_{c}$ limit, we may discard all but the first 
four terms if we choose to work only up to subleading order. Acting on a large- $N_{c}$ baryon state

$$
\begin{gathered}
S^{2}=S(S+1), \\
T^{8}=\left(N_{c}-3 N_{s}\right) / \sqrt{12},
\end{gathered}
$$

and for totally-symmetric spin-flavor wave functions

$$
S^{i} G^{i 8}=\frac{1}{4 \sqrt{3}}\left[3 I(I+1)-S(S+1)-3 N_{s}\left(N_{s}+2\right) / 4\right] .
$$

These identities imply that the only effects of the discarded terms up through order $1 / N_{c}$ are redefinitions of the first four coefficients, $a_{1} \ldots a_{4} \rightarrow a_{1}^{\prime} \ldots a_{4}^{\prime}$. Thus we may write the mass eigenvalues

$$
\begin{aligned}
M=a_{1}^{\prime} N_{c} & +\frac{a_{2}^{\prime}}{N_{c}} S(S+1)+\epsilon a_{3}^{\prime}\left(N_{c}-3 N_{s}\right) \\
& +\epsilon \frac{a_{4}^{\prime}}{2 \sqrt{12} N_{c}}\left[3 I(I+1)-S(S+1)-3 N_{s}\left(N_{s}+2\right) / 4\right]+\mathcal{O}\left(1 / N_{c}^{2}\right) .
\end{aligned}
$$

Eq. (3.1) then follows from a trivial redefinition of the coefficients. In modern language, the $\mathrm{SU}(6)$ breaking representations that lead to the GR mass formula are precisely those that arise at leading and subleading order in the $1 / N_{c}$ expansion.

Using the observed masses in Table [, we may solve for the coefficients in Eq. (3.1). In units of $\mathrm{MeV}$, we find

$$
\begin{array}{ll}
A=1406.3 \pm 31.3, & B=195.0 \pm 43.0 \\
C=15.0 \pm 38.1, & D=43.3 \pm 46.0
\end{array}
$$

from which we predict the following states:

$$
\Xi^{\prime}(1825 \pm 98), \quad \Sigma^{* \prime}(1790 \pm 192), \quad \Xi^{*^{\prime}}(1955 \pm 196), \quad \Omega^{\prime}(2120 \pm 234) .
$$

\section{DECAY WIDTH PREDICTIONS}

From Eq. (1.3), the decay width is given by

$$
\Gamma=\frac{M_{f}}{6 \pi M_{i}} k^{2} f(k)^{2}|\mathcal{G}|^{2}
$$

where $M_{i}$ and $M_{f}$ are the masses of the initial and final baryons and

$$
|\mathcal{G}|^{2} \equiv \sum\left|\left\langle B_{f}\left|G_{j a}\right| B_{i}\right\rangle\right|^{2}
$$

where the sum is over final state spins and isospins. The quantity $|\mathcal{G}|^{2}$ may be obtained either by use of symbolic math code [9] or by using 


$$
\left\langle B_{f}\left|G_{j a}\right| B_{i}\right\rangle=\left\langle B_{f}\left(I_{f}, \alpha_{f}, S_{f}, m_{f}\right)\left|G_{j a}\right| B_{i}\left(I_{i}, \alpha_{i}, S_{i}, m_{i}\right)\right\rangle=\mathcal{G}\left(\begin{array}{cc|c}
I_{f} & I_{a} & I_{i} \\
\alpha_{f} & \alpha_{a} & \alpha_{i}
\end{array}\right)\left(\begin{array}{cc|c}
S_{f} & 1 & S_{i} \\
m_{f} & j & m_{i}
\end{array}\right)
$$

and evaluating the left-hand side for one particular final state. Here, $\alpha$ and $m$ stand for isospin and spin projections, and the sum in equation (4.2) is over $\alpha_{f}, \alpha_{a}, m_{f}$, and $j$.

We have already discussed fitting the function $f(k)$ to the measured decays, and how to use the Gürsey-Radicati mass formula to obtain the masses of the unobserved $\mathbf{5 6}^{\prime}$ states. Using the results of the fit with $f(k) \propto 1 / k$, we predict the remainder of the strong decays

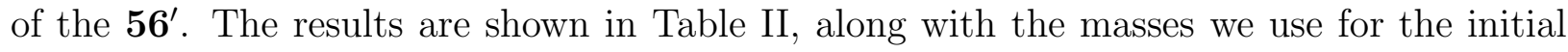
baryons.

Table II does not quote the uncertainties for the widths. Numerical uncertainties come from two sources. One is the uncertainty in the function $f(k)$, and the other is the uncertainty in the masses. The uncertainty in the fitted $f(k)$ leads to about $\pm 15 \%$ uncertainties in the widths. Further width uncertainty induced by mass uncertainty can be large, as discussed below. Of course, once the state is found, the widths in Table II can be recalculated easily and accurately with the correct mass.

\section{DISCUSSION}

The uncertainty in predicting the width of the $\mathbf{5} \mathbf{6}^{\prime}$ decays is dominated not by uncertainty in the matrix elements but by uncertainty in the masses of the states. Four of the $\mathbf{5 6}^{\prime}{ }^{\prime}$ masses are measured and four are not. We must predict the unmeasured masses, and do so using the Gürsey-Radicati mass formula. The accuracy of masses predicted from the Gürsey-Radicati formula is ultimately limited by the the approximations inherent in its derivation, and is easily estimated in a large- $N_{c}$ scheme. Currently, however, uncertainties in the predicted masses are dominated by uncertainties in the measured masses, and they are not small.

Table \1 shows the predicted partial width of the various decay modes, using the central values of the predicted masses. To help see how the mass uncertainties affect us, consider the equal spacing rule that follows from the GR formula. The decuplet spacing is given by

$$
\Delta m_{10^{\prime}}=\frac{3}{2} m\left(\Lambda^{\prime}\right)-\frac{1}{2} m\left(\Sigma^{\prime}\right)-m\left(N^{\prime}\right)=(165 \pm 110) \mathrm{MeV}
$$

(For comparison, the equivalent prediction for the ground state decuplet is $\Delta m_{10}=139 \mathrm{MeV}$, to be compared to 153,148 , and $139 \mathrm{MeV}$ for the $\Delta-\Sigma^{*}, \Sigma^{*}-\Xi^{*}$, and $\Xi^{*}-\Omega$ mass splittings, respectively.) Then, as one example, the predicted central value for the $\Omega^{\prime}$ mass is $2.12 \mathrm{GeV}$, and this leads to a sizeable decay width for $\Omega^{\prime} \rightarrow \Xi^{*} \bar{K}$. However, the uncertainties allow the $\Omega^{\prime}$ to lie below the threshold for this decay.

Moreover, a simple weighted averaging of the published results that the RPP [14] uses as the basis of their mass estimates for the $\Lambda^{\prime}$ and $\Sigma^{\prime}$ gives somewhat different central masses and much tighter uncertainties than they conservatively quote. One finds

$$
\begin{gathered}
m\left(\Lambda^{\prime}\right)=(1600 \pm 7) \mathrm{MeV} \\
m\left(\Sigma^{\prime}\right)=(1672 \pm 5) \mathrm{MeV}
\end{gathered}
$$


(The error limit on the $\Lambda^{\prime}$ mass includes a scale factor $S=1.4$, in accordance with procedures described in the RPP narrative.) Using these masses together with the previous $N^{\prime}$ and $\Delta^{\prime}$ masses yields

$$
\Delta m_{10^{\prime}}=(114 \pm 20) \mathrm{MeV}
$$

This reduced spacing leads to a number of decays listed in Table II being kinematically forbidden.

Unfortunately, there is not much one can do theoretically to reduce the uncertainties originating from the $\mathbf{5 6 ^ { \prime }}$ masses. Table $\mathbb{\text { I }}$ simply presents the most reliable predictions we can make with the current data. As unobserved states are discovered, and their masses measured, one can easily revise the widths in Table [1]. To reiterate, the relevant formula for our $k f(k)=$ const. $=a$ fit is

$$
\Gamma=\frac{k M_{f}}{6 \pi M_{i}} a^{2}|\mathcal{G}|^{2},
$$

where $M_{i}, M_{f}$, and $k$ are defined already, the matrix element sum $|\mathcal{G}|^{2}$ is given in Table 【, and $a$ is $2.8 \pm 0.20$, based on the current data.

The function $f(k)$ is not taken from any model, but fit to the limited data with the fulfilled hope that there would be a good fit using a simple form. The form one would expect from an atomic physics calculation is rather different from the one we found. For the hydrogen atom, in a nonrelativistic calculation, $f(k)$ would be proportional to a matrix element of $\exp (i \vec{k} \cdot \vec{r})$ between $2 \mathrm{~S}$ and $1 \mathrm{~S}$ wave functions. Further in a hydrogen atom, the wavelength of the outgoing radiation is long compared to the Bohr radius, and the small $k$ result that $f(k) \propto k^{2}$ is valid. This leads to a very small width and the famous metastability of the hydrogen $2 \mathrm{~S}$ state. For particle decay, the wavelength of the outgoing meson is comparable to and often smaller than the size scale of the baryon states. One should have no expectation that a low $k$ approximation will work. This also applies to barrier factors in the non-metastable case.

A model or an eventual ab initio calculation for the baryon states will produce some definite $f(k)$ that may or may not look analytically like our form, but to fit the data must not be numerically dissimilar to our result.

To summarize, we have without reference to models, but using the limited available data, studied the strong decays of the lowest-lying radially excited baryons. We assumed, justified by previous studies, that one-body operators dominated and that configuration mixing could be neglected. Simple forms for the one momentum-dependent function give a good fit to existing data, and allow prediction of 22 additional decay modes for this multiplet.

\section{Acknowledgments}

We thank R. Horgan for useful communications, and R. F. Lebed for a careful reading of the manuscript. CDC and CEC thank the National Science Foundation for support under Grant No. PHY-9900657. In addition, CDC thanks the National Science Foundation for support under grant No. PHY-9800741 and the Jeffress Memorial Trust for support under Grant No. J-532. 


\section{REFERENCES}

[1] F. Gürsey and L. Radicati, Phys. Rev. Letters 13 (1964) 173.

[2] Zhen-ping Li, V. Burkert, and Zhu-jun Li, Phys. Rev. D 46, 70 (1992); C. E. Carlson and N. C. Mukhopadhyay, Phys. Rev. Lett. 67, 3745 (1991).

[3] O.Krehl, C.Hanhart, S.Krewald, and J.Speth, nucl-th/9911080.

[4] N. Isgur and G. Karl, Phys. Rev. D 19, 2653 (1979).

[5] L. Ya. Glozman and D.O. Riska, Phys. Rept. 268, 263 (1996).

[6] Shoichi Sasaki, et al., Invited Talk at NSTAR2000, The Physics of Excited Nucleons, Newport News, VA, 16-19 February 2000, hep-ph/0004252.

[7] A.J.G Hey, P.J. Litchfield and R.J. Cashmore, Nucl. Phys. B 95 (1975) 516; R. Horgan, Proceedings of the Topical Conference on Baryon Resonances, Oxford, (1976) 435.

[8] C.E. Carlson, C.D. Carone, J.L Goity and R.F. Lebed, Phys. Lett. B 438 (1998) 327; Phys. Rev. D 59 (1999) 114008.

[9] C.D. Carone, H. Georgi, L. Kaplan, and D. Morin, Phys. Rev. D 50 (1994) 5793.

[10] C.E. Carlson and C.D. Carone, Phys. Rev. D 58 (1998) 053005; Phys. Lett. B 441 (1998) 363.

[11] D. Pirjol and T-M Yan, Phys. Rev. D 57 (1998) 1449; ibid. 5434.

[12] N. Isgur and G. Karl, Phys. Rev. D 19 (1979) 2653.

[13] A. J. Buchmann, R. F. Lebed, hep-ph/0003167.

[14] Particle Data Group (C. Caso et al.), Eur. Phys. J. C 3, 1 (1998).

[15] M. Beg and V. Singh, Phys. Rev. Letters 13 (1964) 418.

[16] E. Jenkins and R. F. Lebed, Phys. Rev. D 52 (1995) 282; A. Manohar, e-print archive: hep-ph/9802419. 


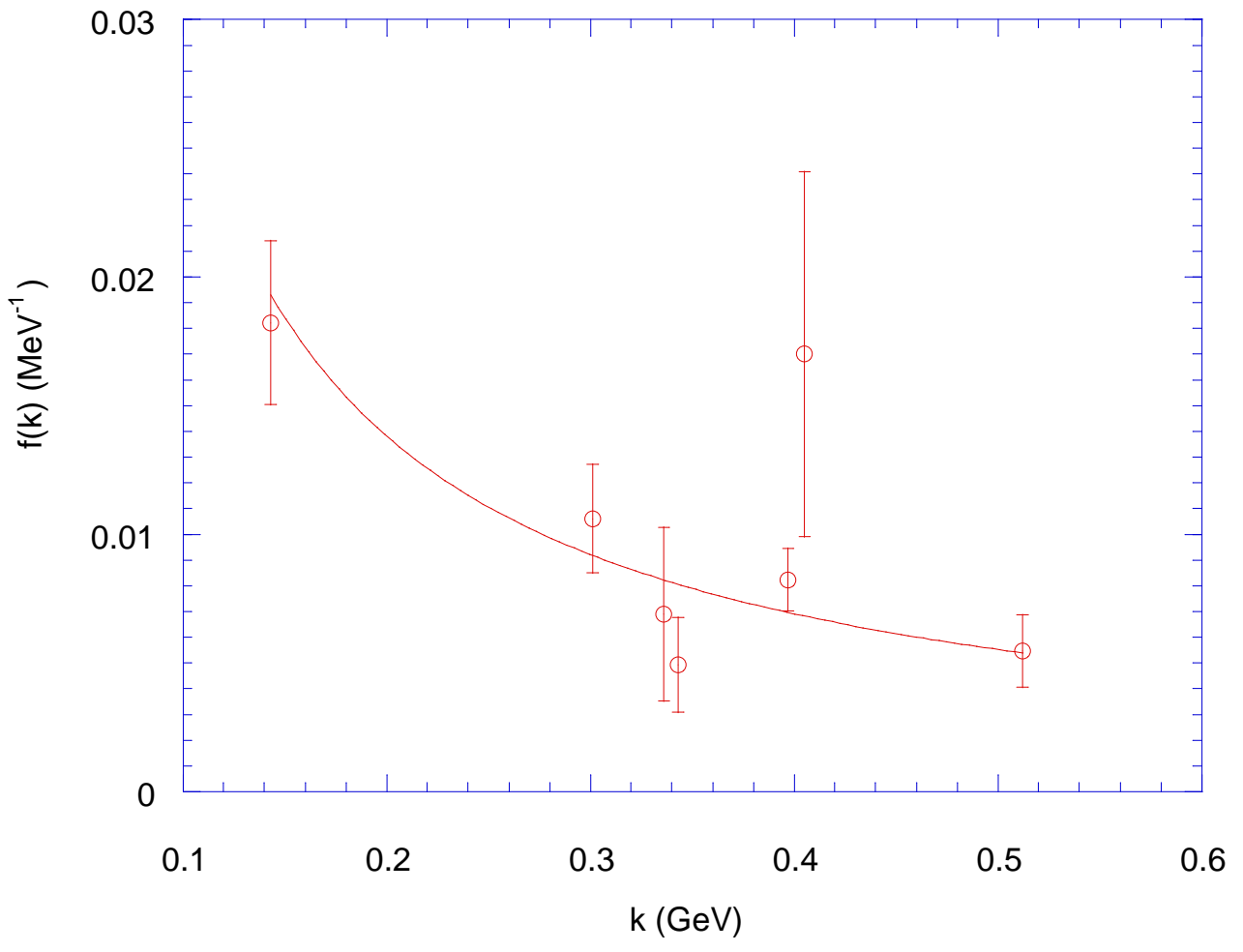

FIG. 1. The function $f(k)$. The best fit corresponds to $f=2.8 / k$, with $\chi^{2} /$ d.o.f $=1.1$. 


\section{TABLES}

\begin{tabular}{lcccc}
\hline \hline & Mass $(\mathrm{MeV})$ & Decay & $k(\mathrm{MeV})$ & Partial Width (MeV) \\
\hline$N(1440)$ & $1450 \pm 20$ & $N \pi$ & 397 & \\
& & $\Delta \pi$ & 143 & $227.5 \pm 67.3$ \\
& & $N \pi$ & 512 & $87.5 \pm 30.5$ \\
\hline$\Delta(1600)$ & $1625 \pm 75$ & $3 \pi$ & 301 & $61.25 \pm 31.5$ \\
& & & 343 & $192.5 \pm 76.0$ \\
\hline$\Lambda(1600)$ & $1630 \pm 70$ & $N \bar{K}$ & 336 & $33.75 \pm 25.2$ \\
& & $\Sigma \pi$ & & $52.5 \pm 51.3$ \\
\hline$\Sigma(1660)$ & $1660 \pm 30$ & $N \bar{K}$ & 405 & $24.0 \pm 20.0$ \\
\hline \hline
\end{tabular}

TABLE I. Input values used in the determination of $f(k)$. 


\begin{tabular}{|c|c|c|c|c|}
\hline Decay & $M_{i}(\mathrm{MeV})$ & $k(\mathrm{MeV})$ & $|\mathcal{G}|^{2}$ & $\Gamma(\mathrm{MeV})$ \\
\hline$N^{\prime} \rightarrow N \pi^{\dagger}$ & 1450 & 397 & $25 / 16$ & 164 \\
\hline$N^{\prime} \rightarrow \Delta \pi^{\dagger}$ & 1450 & 143 & 2 & 99 \\
\hline$\Delta^{\prime} \rightarrow N \pi^{\dagger}$ & 1625 & 512 & $1 / 2$ & 60 \\
\hline$\Delta^{\prime} \rightarrow \Delta \pi^{\dagger}$ & 1625 & 301 & $25 / 16$ & 145 \\
\hline${\overline{\Lambda^{\prime}} \rightarrow N \bar{K}^{\dagger}}^{\dagger}$ & 1630 & 343 & $9 / 8$ & 91 \\
\hline$\Lambda^{\prime} \rightarrow \Sigma \pi^{\dagger}$ & 1630 & 336 & $3 / 4$ & 75 \\
\hline$\Lambda^{\prime} \rightarrow \Sigma^{*} \pi$ & 1630 & 188 & $3 / 2$ & 97 \\
\hline${\overline{\Sigma^{\prime}} \rightarrow N \bar{K}^{\dagger}}^{\dagger}$ & 1660 & 405 & $1 / 24$ & 4 \\
\hline$\Sigma^{\prime} \rightarrow \Lambda \pi$ & 1660 & 439 & $1 / 4$ & 30 \\
\hline$\Sigma^{\prime} \rightarrow \Sigma \pi$ & 1660 & 385 & $2 / 3$ & 75 \\
\hline$\Sigma^{\prime} \rightarrow \Sigma^{*} \pi$ & 1660 & 218 & $1 / 3$ & 25 \\
\hline$\Xi^{\prime} \rightarrow \Lambda \bar{K}$ & 1825 & 403 & $1 / 16$ & 6 \\
\hline$\Xi^{\prime} \rightarrow \Sigma \bar{K}$ & 1825 & 323 & $25 / 16$ & 134 \\
\hline$\Xi^{\prime} \rightarrow \Xi \pi$ & 1825 & 420 & $1 / 16$ & 8 \\
\hline$\Xi^{\prime} \rightarrow \Xi^{*} \pi$ & 1825 & 240 & $1 / 2$ & 41 \\
\hline$\overline{\Sigma^{* \prime} \rightarrow N \bar{K}}$ & 1790 & 519 & $1 / 6$ & 18 \\
\hline$\Sigma^{* \prime} \rightarrow \Delta \bar{K}$ & 1790 & 214 & $5 / 6$ & 50 \\
\hline$\Sigma^{* \prime} \rightarrow \Lambda \pi$ & 1790 & 535 & $1 / 4$ & 34 \\
\hline$\Sigma^{* \prime} \rightarrow \Sigma \pi$ & 1790 & 484 & $1 / 6$ & 22 \\
\hline$\Sigma^{* \prime} \rightarrow \Sigma^{*} \pi$ & 1790 & 338 & $5 / 6$ & 89 \\
\hline$\Sigma^{* \prime} \rightarrow \Sigma \eta$ & 1790 & 196 & $1 / 4$ & 13 \\
\hline$\Xi^{* \prime} \rightarrow \Lambda \bar{K}$ & 1955 & 525 & $1 / 4$ & 31 \\
\hline$\Xi^{* \prime} \rightarrow \Sigma \bar{K}$ & 1955 & 461 & $1 / 4$ & 29 \\
\hline$\Xi^{* \prime} \rightarrow \Sigma^{*} \bar{K}$ & 1955 & 239 & $5 / 4$ & 86 \\
\hline$\Xi^{* \prime} \rightarrow \Xi \pi$ & 1955 & 520 & $1 / 4$ & 36 \\
\hline$\Xi^{* \prime} \rightarrow \Xi^{*} \pi$ & 1955 & 358 & $5 / 16$ & 36 \\
\hline$\Xi^{* \prime} \rightarrow \Xi \eta$ & 1955 & 269 & $1 / 4$ & 19 \\
\hline$\Omega^{\prime} \rightarrow \Xi \bar{K}$ & 2120 & 506 & 1 & 128 \\
\hline$\Omega^{\prime} \rightarrow \Xi^{*} \bar{K}$ & 2120 & 274 & $5 / 4$ & 101 \\
\hline
\end{tabular}

TABLE II. Decays of the radially excited $\mathbf{5 6} \mathbf{6}^{\prime}$ into ground state $\mathbf{5 6}$ plus meson. Seven of these decays are measured (indicated by a dagger), and the other 22 widths are predictions. If the mass of the initial state is known, the uncertainty in the width from our fitting procedure is $\pm 15 \%$. Uncertainties in the mass induce further and sometimes large uncertainties in the width. These of course are greatly reducible once the mass of the state is measured, as discussed in the text. 